- Case Report

\title{
Olfactory Function Test for Early Diagnosis of Vascular Dementia
}

\author{
Kang Duk Suh', Sun Mi Kim², Doug Hyun Han², Hyun Jin Min', Kyung Soo Kim ${ }^{1, *}$ \\ 'Department of Otorhinolaryngology-Head and Neck Surgery, Chung-Ang University College of Medicine, Seoul, Korea \\ ${ }^{2}$ Department of Psychiatry, Chung-Ang University College of Medicine, Seoul, Korea
}

Olfactory impairment occurs in patients with Alzheimer's disease, and olfactory function tests are performed for the diagnosis of Alzheimer's disease. However, the diagnosis and patient status are not currently outlined for vascular dementia, and many physicians do not consider concurrent vascular dementia in patients complaining of olfactory dysfunction. Here, we report a case of vascular dementia with no symptoms of dementia other than olfactory dysfunction. This case suggested that the olfactory function test is helpful not only for the diagnosis of Alzheimer's disease but also for the early diagnosis of vascular dementia.

Keywords: Olfactory Dysfunction; Vascular Dementia; Diagnosis; Olfactory Function Test 


\section{INTRODUCTION}

Olfactory dysfunction may occur in the course of natural aging but may also be accompanied by neurodegenerative diseases, such as dementia. There are various subtypes of dementia, including Alzheimer's dementia, vascular dementia, dementia in Parkinson's disease, and frontotemporal dementia. ${ }^{1)}$ Alzheimer's disease is characterized by memory loss, which is associated with neuropathological changes, such as neurofibrillary tangles, neurotic plaques, and atrophy. ${ }^{1)}$ Vascular dementia is characterized by cognitive decline and concomitant cerebrovascular disease. ${ }^{2)}$ Parkinson's disease is characterized by movement disorder and loss of nigrostriatal dopaminergic neurons. Frontotemporal dementia is characterized by atrophy and frontal and anterior temporal lobe degenerations. ${ }^{2)}$ Multiple studies showed a relationship between dementia and olfactory dysfunction. Olfactory dysfunction, particularly odor identification deficit, is a symptom of Alzheimer's disease; olfactory symptoms may occur in the mild cognitive impairment stage. ${ }^{3)}$ In Parkinson's disease, $80 \%-90 \%$ of patients complain of olfactory dysfunction, and these symptoms occur before the appearance of motor symptoms. ${ }^{3)}$ Olfactory dysfunction may be present in frontotemporal dementia but is not as common as in Parkinson's disease. ${ }^{4)}$

However, studies on olfactory function in vascular dementia are limited. In addition, the results are inconsistent across studies, which further limit the applications for research and clinical practice. Here, we report a case of vascular dementia in a patient who complained of olfactory dysfunction.

\section{CASE REPORT}

A 75-year-old man was referred to Chung-Ang University Hospital because of anosmia for 3 months. He had no medical or psychological diseases. His nasal septum was deviated to the left, and nasal endoscopy and paranasal sinus computed tomography showed no other abnormal findings. Serologic examination for the levels of vitamin $B_{12}$, zinc, and copper were unremarkable. An olfactory function test, which is the Korean version of Sniffin' Sticks test, KVSS II, ${ }^{5)}$ was to assess the olfactory threshold, odor discrimination, and odor identification (Transition Dyspnea Index [TDI] score). The total TDI score was 11 (threshold, 0; discrimination, 6; identification, 5), and the patient was diagnosed with anosmia, which was unresponsive to medical treatment, including oral methylprednisolone, pseudoephedrine, and mometasone nasal spray. Although he had no complaints related to cognitive function, we referred him to a neuropsychiatry clinic to evaluate the possibility of concurrent central nervous system abnormalities. The Mini-Mental State Examination (MMSE) and Clinical Dementia Rating (CDR) were performed. The MMSE score was within the normal range, and the CDR score was 1 , which was indicative of relevant to mild cognitive impairment. Additionally, brain magnetic resonance imaging (MRI) was performed, and multiple ischemic changes in bilateral periventricular white matter and minimal brain atrophy were observed (Figure 1). Although the MMSE score was within the normal range, vascular dementia was diagnosed based on MRI findings, clinical history, and the CDR score. The patient was prescribed with donepezil for dementia treatment and is currently undergoing regular follow-up with olfactory training.

The patient provided written informed consent for the publication of clinical details and images.

\section{DISCUSSION}

Patients with clinically diagnosed Alzheimer's disease show a marked impairment in their ability to identify odors. The prevalence of olfactory dysfunction is estimated to be as high as $100 \%$ in Alzheimer's disease ${ }^{6)}$ However, olfactory impairment in vascular dementia is less clear. Olfactory abnormalities occur in patients with multiple infarct dementia but are not so pronounced as in Alzheimer's disease. ${ }^{7)}$ All stroke patients are normosmic or only slightly hyposmic. ${ }^{8)}$ In a study, the prevalence of olfactory dysfunction in vascular dementia was approximately $15 \%{ }^{6}{ }^{6}$ However, in another study, patients with vascular dementia showed a similar level of olfactory identification ability as patients with Alzheimer's disease. ${ }^{7)}$

Vascular dementia is characterized by cognitive decline, typically in a stepwise manner, compatible with dementia related to cerebrovascular disease. ${ }^{1,9)}$ The mechanism of olfactory impairment in patients with vascular dementia is unclear, as the distribution of cerebral pathological changes does not usually affect structures involved in olfaction. It is hypothesized that the presence/absence, range, extent, and type of olfactory deficits may be influenced by the location and extension of vascular pathology. ${ }^{1)}$

It is important to differentiate vascular dementia from other types of dementia and diagnose it in the prodromal stage because early diag-

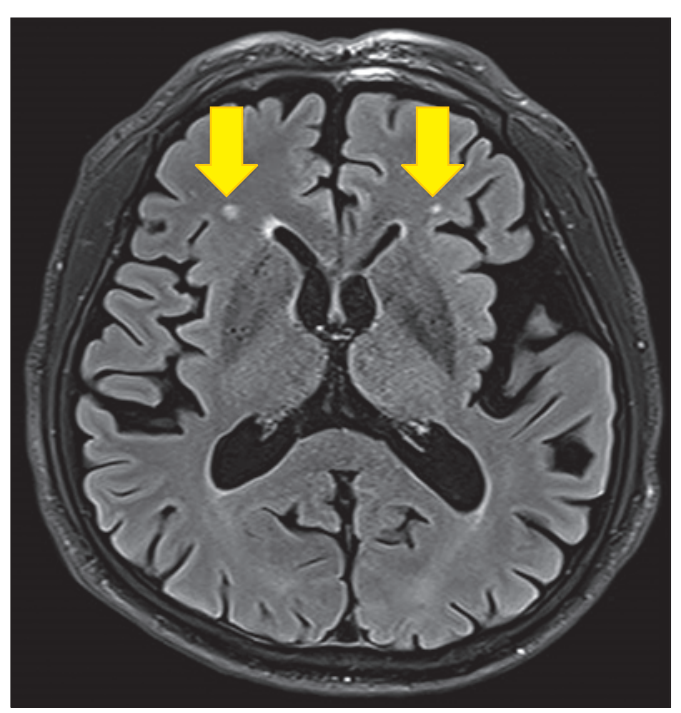

Figure 1. The brain magnetic resonance imaging scan shows multiple ischemic changes in bilateral periventricular white matter (yellow arrows) and minimal brain atrophy. 
nosis with early preventive intervention can delay the progression of the disease, as in cases of vascular dementia. ${ }^{10)}$ However, the possibility of vascular dementia is frequently ignored, and evaluation is often not performed. Vascular pathological conditions in early stages of vascular dementia may present with various systemic symptoms, such as swallowing disorders, walking anxiety, frequent falls, personality and mood disorders, and when these symptoms manifest, patients are generally diagnosed by specialized medical staff, delaying the early diagnosis of vascular dementia. ${ }^{10)}$ As vascular dementia can present with varying systemic symptoms, depending on the vascular lesion site and extent, and as in this case, olfactory dysfunction could be considered as a systemic symptom that could occur in the early stages of vascular dementia. A smell test is a useful tool for distinguishing among Alzheimer's disease, vascular dementia, and major depression. ${ }^{6}$

This case suggests that old patients with complaints of olfactory disorder but without symptoms of cognitive decline should be assessed further. Therefore, the olfactory function test could be useful for early detection of underlying vascular dementia, which could be evaluated using active questionnaires and imaging studies.

\section{CONFLICT OF INTEREST}

No potential conflict of interest relevant to this article was reported.

\section{ACKNOWLEDGMENTS}

This study was supported by the Biomedical research institute of the Chung-ang University Hospital.

\section{ORCID}

Sun Mi Kim: https://orcid.org/0000-0003-4131-0542 Doug Hyun Han: https://orcid.org/0000-0001-5888-0686

Hyun Jin Min: https://orcid.org/0000-0003-3075-1350
Kyung Soo Kim: https://orcid.org/0000-0003-2637-0555

\section{REFERENCES}

1. Alves J, Petrosyan A, Magalhães R. Olfactory dysfunction in dementia. World J Clin Cases 2014;2:661-7.

2. Bouchard RW. Diagnostic criteria of dementia. Can J Neurol Sci 2007;34 Suppl 1:S11-8.

3. Fusetti M, Fioretti AB, Silvagni F, Simaskou M, Sucapane P, Necozione S, et al. Smell and preclinical Alzheimer disease: study of 29 patients with amnesic mild cognitive impairment. J Otolaryngol Head Neck Surg 2010;39:175-81.

4. Heyanka DJ, Golden CJ, McCue RB 2nd, Scarisbrick DM, Linck JF, Zlatkin NI. Olfactory deficits in frontotemporal dementia as measured by the Alberta Smell Test. Appl Neuropsychol Adult 2014;21:176-82.

5. Cho JH, Jeong YS, Lee YJ, Hong SC, Yoon JH, Kim JK. The Korean version of the Sniffin' stick (KVSS) test and its validity in comparison with the cross-cultural smell identification test (CC-SIT). Auris Nasus Larynx 2009;36:280-6.

6. Duff K, McCaffrey RJ, Solomon GS. The Pocket Smell Test: successfully discriminating probable Alzheimer's dementia from vascular dementia and major depression. J Neuropsychiatry Clin Neurosci 2002;14:197-201.

7. Gray AJ, Staples V, Murren K, Dhariwal A, Bentham P. Olfactory identification is impaired in clinic-based patients with vascular dementia and senile dementia of Alzheimer type. Int J Geriatr Psychiatry 2001;16:513-7.

8. Cecchini MP, Bojanowski V, Bodechtel U, Hummel T, Hahner A. Olfactory function in patients with ischemic stroke: a pilot study. Eur Arch Otorhinolaryngol 2012;269:1149-53.

9. Wiederkehr S, Simard M, Fortin C, van Reekum R. Validity of the clinical diagnostic criteria for vascular dementia: a critical review: part II. J Neuropsychiatry Clin Neurosci 2008;20:162-77.

10. Jaul E, Meiron O. Systemic and disease-specific risk factors in vascular dementia: diagnosis and prevention. Front Aging Neurosci 2017;9:333. 